\title{
Sero-epidemiology of Plasmodium vivax infection among Sudanese's and Ethiopians in Khartoum State
}

\begin{abstract}
Background: Plasmodium vivax is most widely distributed in the world, and to be considered absent from western Africa, where the prevalence of Duffy negative red blood cell phenotype proves to be high.
\end{abstract}

Objectives: This study aimed at determines the prevalence of Plasmodium vivax among Sudanese and Ethiopians individuals in relation to hematological parameter effect.

Methods: This was across-sectional descriptive study involving 1200 individual (600 Sudanese and 600 Ethiopians) form different age groups during the period June 2015 to February 2018.

Results: The prevalence of Plasmodium vivax among 1200 individuals, of whom 600 were Sudanese and 600 were Ethiopians subjected to screening by ICTs and blood films tests, showed that $100(8.3 \%)$ gave positive results, out of them $(2.08 \%)$ Sudanese whereas were $(6.25 \%)$ became Ethiopians survived population were divided in to five age groups (5$15),(15-25),(25-35)$ and( more than 35 years). The positive case within each age groups showed $(13.7 \%),(1.1 \%),(.71 \%)$ and $(0.0 \%)$ respectively among Sudanese and $(2.8 \%)$, $(13.6 \%),(10 \%)$ and $(27.3 \%)$ respectively among Ethiopians. The results showed the hematological effected among Sudanese groups, 96\% were thrombocytopenia, $80 \%$ leucopenia and $88 \%$ anemia. Other parameter $\mathrm{MCV}(48 \%), \mathrm{MCH}(60 \%)$, and $\mathrm{MCHC}(70 \%)$. Where were $70 \%$ thrombocytopenia, $40 \%$ leucopenia and $53 \%$ anemia, MCV (16\%), MCH (26\%) and MCHC (33\%) among Ethiopian groups.

Conclusion: The sero-prevalence of Plasmodium vivax is relatively low among Sudanese than Ethiopian. Thrombocytopenia, anemia and leucopenia is most frequency in Plasmodium vivax infection.

Keywords: prevalence, Plasmodium vivax, malaria, thrombocytopenia, leucopenia, anemia

\section{Introduction}

Plasmodium vivax is a hemo-pathogenic parasite that belongs for the phylum Apicomplexa. It causes malaria which is the public health significant. Most of the research and published literature on malaria focuses on Plasmodium falciparum and much less on Plasmodium vivax. ${ }^{1}$ This focus is due to the very high burden of mortality attributed to the falciparum species in Africa. ${ }^{2}$ However, there is growing evidence that Plasmodium vivax is responsible for a significant burden of disease worldwide accounting for half of all malaria cases in Asia and Latin America, will nearly 2.5 billion people at risk of infection. ${ }^{3}$ All species are transmitted by the bite of an infective female Anopheles mosquitoes control and management of vivax malaria is difficult, and the present particular challenges. ${ }^{4}$ Hematological changes that have been reported to accompany malaria are anemia, thrombocytopenia, leucopenia, atypical lymphocytosis, and infrequently disseminated intravascular coagulation. ${ }^{5}$ Some studies have shown that Plasmodium vivax can also cause severe disease and the hematological parameters are usually changed in such cases. The present study was undertaken to assess the hematological alterations in vivax malaria patients in comparison between Sudanese and Ethiopians. ${ }^{6}$

\section{Materials and methods}

\section{The study area and population}

The study was conducted in three Sudanese states (Blue Nile State, White Nile state and Khartoum State) among infected with Plasmodium vivax Sudanese and Ethiopians.

\author{
Volume 7 Issue 5 - 2019
}

\author{
Mohanad E Alfadol,' Mohammed Medani \\ Eltayeb, ${ }^{2}$ Mosab Nouraldein Mohammed \\ Hamad $^{3}$ \\ 'Department of Parasitology, Faculty of Medical Laboratory \\ Sciences, Omdurman Islamic University, Sudan \\ ${ }^{2}$ Department of Medical Parasitology, Faculty of Medical \\ Laboratory Sciences, Alneelain University, Sudan \\ ${ }^{3}$ Department of Medical Parasitology, Faculty of Health Sciences, \\ Elsheikh Abdallah Elbadri University, Sudan
}
Correspondence: Mosab Nouraldein Mohammed Hamad, Department of Medical Parasitology, Faculty of Health Sciences, Elsheikh Abdallah Elbadri University, Sudan, Emailmusab.noor13@gmail.com

Received: August 09, 2019 | Published: September 26, 2019

\section{Sample size}

The minimum sample size for the study was 1200 patients calculated by using the formula

\section{Data collection and analysis}

The study participants were interviewed by administration of stander questionnaire to obtain the socio-demographic and economic status information as well as epidemiological risk factors. Laboratory results from the test were entered using the same number as the one on the questionnaires. All the data were entered in to excel spread sheet and the later exported in to SPSS for statistical analysis.

\section{Sample collection}

Venous blood sample collected randomly in EDTA container $(2,5$ $\mathrm{mL}$ ), About 4 drops form blood was put on slide to make thin and thick blood film $20 \mu \mathrm{m}$ examine by used ICT test and $2 \mathrm{~mL}$ to deterrent the hematological parameter value.

\section{Results and discussion}

In this study the prevalence of Plasmodium vivax infection among Sudanese and Ethiopian of different age groups of both male and female was compared by using various techniques. The overall prevalence rate among the study groups was $8.33 \%$, The prevalence in Sudanese was $2.08 \%$ which is similar to the results obtained by WHO (2011) who found that the rate of Plasmodium vivax in children in New Halfa was (3\%) $.1 .5 \%$ in Kassala as reported by (Mamoun 
et al., 2016), and 35\% reported by (Tarig et al., 2015) in Khartoum state. The prevalence of Plasmodium vivax in other countries was higher. For example it was $23.8 \%$ in Rural Amazonia (Barbosa et al. , 2010), 13.2\% in Pakistan (Bin et al., ,2014), 81\% in India reported by (WHO 2014), 2.9\% it reported in Solomon Island (Harris et al., 2010), 83(28.7\%) in India by Mayuri et al., 2013, and other studies by Kashinkunti et al., $2013(50 \%)$ in Tertiary Care Centre of North Karnataka. The results obtained showed that the prevalence rate in Ethiopians was $6.25 \%$, such rate is higher than in Sudanese It is difficult to explain the higher prevalence of Plasmodium vivax in Ethiopian however one explanation could be that they came from Ethiopia carrying dormant stage (hypnozoites ) in their livers which upon relapse development malaria . Factors Such as Hereditary or environmental factors or more exposure in Sudan may affect their susceptibility compared to Indians.

Table I Prevalence of Plasmodium vivax infection among the Sudanese and Ethiopians

\begin{tabular}{llll}
\hline Tested individuals & No of examined & No of positive & Prevalence (\%) \\
\hline Sudanese & 600 & 25 & $2.08 \%$ \\
Ethiopian & 600 & 75 & $6.25 \%$ \\
Total & 1200 & 100 & $8.30 \%$ \\
\hline
\end{tabular}

Table 2 Plasmodium vivax infection rate tested by rapid diagnostic tests and blood films in both study groups

\begin{tabular}{llll}
\hline Tested & No examined & No of positive (\%) & No of negative (\%) \\
\hline Blood film & 1200 & $100(8.33 \%)$ & $1100(91.6 \%)$ \\
ICT & 1200 & $70(5.83 \%)$ & $1130(94.2 \%)$ \\
\hline
\end{tabular}

Table 3 Infection rate according to gender in both Sudanese and Ethiopians

\begin{tabular}{lllll}
\hline Gender & No of Sudanese & No of positive (\%) & No of Ethiopian & No of positive (\%) \\
\hline Males & 325 & $15(4.6 \%)$ & 315 & $50(15.8 \%)$ \\
Females & 275 & $10(3.6 \%)$ & 285 & $25(9.6 \%)$ \\
Total & 600 & $15(2.5 \%)$ & 600 & $75(12.5 \%)$ \\
\hline
\end{tabular}

Table 4 The percentage of infection among Sudanese and Ethiopians according to age groups

\begin{tabular}{lll}
\hline Age groups & No of Sudanese positive (\%) & No of Ethiopians positive (\%) \\
\hline I5-May & $22(13.7 \%)$ & $5(2.8 \%)$ \\
$15-25$ & $2(1.1 \%)$ & $20(13.6 \%)$ \\
$25-35$ & I (.71\%) & $15(10 \%)$ \\
More than 35 & $0(0 \%)$ & $35(27.3 \%)$ \\
\hline
\end{tabular}

To compression between blood film and ICT they found that out 1200 sample examined by ICT, 70 samples were positive and the same sample examined by blood film showed 100 samples were positive. The results similar the studies obtained by Mohamed et al., 2016 in Gezira who found, 79.4\%were positive by microcopy and $85.1 \%$ postive by ICT. The infection according to gender showed that it was higher in males of both Sudanese $(4.62 \%)$ and Ethiopians (15.87\%) than females respectively $3.64 \%$ and $8.77 \%$ due to exposure to risk factors outdoor activities such difference among gender may be related to the fact that they usually sleep outside the room and secondary they don't cover themselves properly and This may make them more exposed to mosquitoes. On the other hand women usually stay or sleep inside room a matter that reduces their chance of acquiring infection by mosquitoes. This agrees with study by Kashinkunti et al., 2013 in North Karnataka who showed that out of 95 patients, $50(50.6 \%)$ were infected with Plasmodium vivax, as positives $29(58 \%)$ were males and $21(42 \%)$ were females. The results according to age groups showed that the infection in 5-15 years age individuals $(13.75 \%)$ was higher than $15-25$ years $(1.11 \%)$ and $25-35$ years $(.71 \%)$ in Sudanese this could probably be explanted that the higher rate in young children might be due to low immunity to infection by Plasmodium vivax and not completely covering their bodies during the night.

Among Ethiopians was it most higher in adults in age more than 35 years $(27.66 \%)$ who have more exposure to risk of infection and carrying dormant stage (hypnozoites) in there liver. The results of hematological picture revealed pancytopenia with sever thrombocytopenia (reduction in platelets), as out of 100 positive blood samples, 93\% thrombocytopenia , 40\% leucopenia and $12 \%$ reduce TRBCs. The other parameter in the include $\mathrm{H} \mathrm{b} 47 \%$ indicating anemia, $49 \%$ of MCV, $33 \%$ of $\mathrm{MCH}$ and $\mathrm{MCHC} 37 \%$ from the positive individual. These results agree with the studies done in Khartoum state by (Tarig 2014) who showed that $52.9 \%$ have thrombocytopenia due Plasmodium vivax infection. Other studies obtained by Kashinkunti et al., ( 2013) in Tertiary Care Centre of North Karnataka showed that 24(48\%) were with thrombocytopenia and 33\% with anemia, The results reported by Mayuri et al., (2013) in 
India those $38.6 \%$ were with thrombocytopenia and $89.2 \%$ were with anemia, this study showed higher HB levels and lower in platelets count (thrombocytopenia ). The study done by Bagla J et al.,( 2015) in western Rajasthan in India showed that $44 \%$ were anemic, $71 \%$ with thrombocytopenia and $85 \%$ with leucopenia, these results agree with our the studies on hematological picture. Our explanation of hematological changes is that it is due to the directed effect of hemoglobin synthesis which is reflected on RBCs in their size and shape, A Mechanisms of thrombocytopenia in this study could be due to immune mediated effect on destruction of circulating platelets, decreased platelet survival in vivax malaria.

\section{Conclusion and recommendation}

We conclude that vivax malaria was less common among Sudanese participants in comparison to Ethiopians. Further studies should be done with different diagnostic methods such as molecular techniques (e.g. PCR).

\section{Acknowledgments}

None.

\section{Conflicts of interest}

Authors declare that there is no conflict of interest.

\section{References}

1. Sina B. Focus on Plasmodium vivax. Trends Parasitol.2002;18(7):287-289.

2. Hay S, Guerra C, Tatem A, et al. The global distribution and population at risk of malaria: past, present, and future. Lancet Infect Dis. 2004;4(6):327336.

3. Guerra C, Howes R, Patll A, et al. The international limits and population at risk of Plasmodium vivax transmission. PLoS Negl Trop Dis. 2010;4(8):e774

4. Baird JK. Resistance to therapies for infection by Plasmodium vivax. Clin Microbiol Rev. 2009;22(3):508-534.

5. Facer CA. Hematological aspect of malaria. In: Infection and Hematology. Oxford Butterworth Heinemann Ltd; 1994. 259-294 p.

6. Kueh YK, Yeo KL. Haematological alterations in acute malaria. Scand $J$ Haematol. 2000;29(2):147-152. 